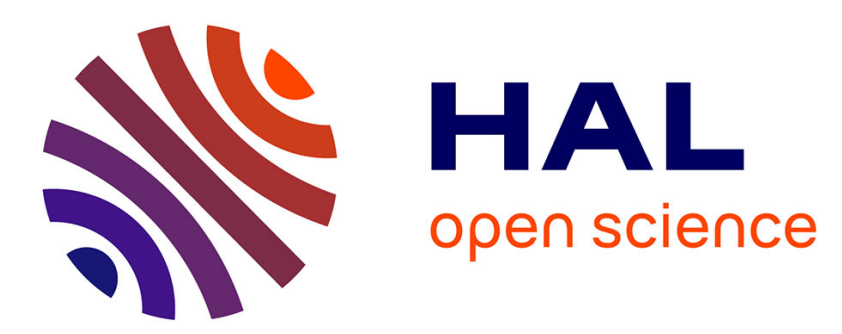

\title{
Choix du coefficient d'interdiffusion régissant le fluage de la phase $\beta$ du Cu-Al (composition eutectoïde)
}

\author{
C. Lexcellent, L. Bornier, J. Birocheau, P. Delobelle
}

\section{To cite this version:}

C. Lexcellent, L. Bornier, J. Birocheau, P. Delobelle. Choix du coefficient d'interdiffusion régissant le fluage de la phase $\beta$ du Cu-Al (composition eutectoïde). Revue de Physique Appliquée, 1985, 20 (8), pp.545-551. 10.1051/rphysap:01985002008054500 . jpa-00245369

\section{HAL Id: jpa-00245369 https://hal.science/jpa-00245369}

Submitted on 1 Jan 1985

HAL is a multi-disciplinary open access archive for the deposit and dissemination of scientific research documents, whether they are published or not. The documents may come from teaching and research institutions in France or abroad, or from public or private research centers.
L'archive ouverte pluridisciplinaire HAL, est destinée au dépôt et à la diffusion de documents scientifiques de niveau recherche, publiés ou non, émanant des établissements d'enseignement et de recherche français ou étrangers, des laboratoires publics ou privés. 


\title{
Choix du coefficient d'interdiffusion régissant le fluage de la phase $\beta$ du Cu-Al (composition eutectoïde)
}

\author{
C. Lexcellent, L. Bornier, J. Birocheau et P. Delobelle \\ Laboratoire de Mécanique Appliquée (*), Faculté des Sciences et des Techniques, \\ La Bouloie, Route de Gray, 25030 Besançon Cedex, France
}

(Reçu le 15 novembre 1984, révisé le 16 avril 1985, accepté le 23 avril 1985)

\begin{abstract}
Résumé. - Grâce à des mesures de fluage, nous effectuons le choix du coefficient de diffusion qui régit le comportement viscoplastique de $\mathrm{CuAl}(\beta)$. Les coefficients d'interdiffusion mesurés par Romig et Pimenov qui conduisent à des résultats significativement différents, sont pris en compte. L'analyse d'Ashby de l'équation de Dorn permet de choisir le coefficient de Pimenov et de relier le comportement en fluage haute température du $\mathrm{CuAl}(\beta)$ à des mécanismes de courts-circuits de diffusion. Notre travail montre que le facteur thermodynamique doit être intégré dans la corrélation fluage-diffusion.
\end{abstract}

\begin{abstract}
With creep's measures, we choose the diffusion coefficient which governs the viscoplastic behaviour of $\mathrm{CuAl}(\beta)$. The interdiffusion coefficients of Romig and Pimenov which give significantly different results, are compared. The Dorn's equation analysis of Ashby allows to choose the Pimenov's coefficient and to rely the high temperature creep behaviour of $\mathrm{CuAl}(\beta)$ with mechanisms of short circuits of diffusion. Our work shows that the thermodynamic factor must be integrated in the correlation creep diffusion.
\end{abstract}

\section{Introduction.}

Le fluage des solutions solides concentrées n'a donné lieu qu'à un nombre réduit d'études expérimentales et pose un certain nombre de problèmes quant à son analyse [1].

Cependant, une synthèse de résultats expérimentaux pour l'étude de différentes phases $\beta$ cubiques centrées (A2) de type $\mathrm{W}$ et stable à haute température a été présentée [2].

Ainsi, nous avons examiné le comportement en fluage des alliages équiatomiques $\mathrm{AgCd}, \mathrm{AgZn} \mathrm{[3],}$ $\mathrm{CuZn}, \mathrm{FeCo} 2 \mathrm{~V}$.

Parmi ces phases $\beta$, le cas du $\mathrm{Cu} 25 \%$ at. Al a lui aussi été étudié [4].

Leur écoulement sous contrainte constante est caractérisé par une zone de fluage stationnaire avec une vitesse $\dot{\varepsilon}_{\mathrm{s}}$ suivie d'une zone de fluage tertiaire conduisant à la rupture.

L'absence de fluage primaire est une caractéristique de telles phases.

De surcroît, les mesures de contraintes internes $\sigma_{i}$

(*) Associé au CNRS. effectuées par différentes méthodes $[5,6]$ donnent des valeurs de $\sigma_{i}$ très faibles indépendamment du champ de contraintes ou de l'intervalle de température pour toutes les phases $\beta$ [7] et en particulier pour le $\mathrm{CuAl}$ $[2,4]$.

Ainsi, la contrainte effective $\sigma^{*}$ peut être définie par $\sigma^{*}=\sigma-\sigma_{i}$ proche, dans notre cas, de la contrainte appliquée $\sigma$.

Les paramètres caractéristiques du fluage, l'énergie d'activation et la sensibilité de la vitesse, définis d'une part en terme de contrainte effective, d'autre part, en terme de contrainte appliquée, s'écrivent :

$$
\begin{array}{ll}
Q=\left(\frac{\partial \operatorname{Ln} \dot{\varepsilon}_{\mathrm{s}}}{\partial\left(-\frac{1}{k T}\right)}\right)_{\sigma} \quad Q^{*}=\left(\frac{\partial \operatorname{Ln} \dot{\varepsilon}_{\mathrm{s}}}{\partial\left(-\frac{1}{k T}\right)}\right)_{\sigma^{*}=\sigma-\sigma_{\mathrm{i}}} \\
m=\left(\frac{\partial \operatorname{Ln} \dot{\varepsilon}_{\mathrm{s}}}{\partial \operatorname{Ln} \sigma}\right)_{T} \quad m^{*}=\left(\frac{\partial \operatorname{Ln} \dot{\varepsilon}_{\mathrm{s}}}{\partial \operatorname{Ln} \sigma^{*}}\right)_{T}
\end{array}
$$

Nous obtenons $Q \simeq Q^{*}$ et $m \simeq m^{*}$, ce qui traduit un effet. négligeable des contraintes internes $\sigma_{\mathbf{i}}$. Finale- 
ment, dans cette étude en fluage stationnaire, la variable de contrainte choisie sera la contrainte appliquée $\sigma$ et l'autre variable d'état, la température $T$. Ces alliages ont des valeurs de $m$ de l'ordre de 3,5 excepté le FeCo2V qui montre un accroissement de cet exposant avec la contrainte.

Afin de prendre en compte chaque paramètre physique du fluage, l'équation semi-empirique de Dorn a été utilisée pour rationaliser les résultats expérimentaux obtenus pour ces différentes phases $\beta$.

Cette équation prend la forme :

$$
\frac{\dot{\varepsilon}_{\mathrm{s}} k T}{D G b}=A\left(\frac{\sigma}{G}\right)^{n}
$$

dans laquelle $\boldsymbol{A}$ et $\boldsymbol{n}$ sont des constantes du matériau considéré, $k T$ et $b$ ont leur signification usuelle, $D$ est un coefficient de diffusion (c'est son choix qui fait principalement l'objet de cette note) et $G$ le module de cisaillement.

Dans l'étude précédente [2] pour le $\mathrm{CuAl}$, le choix correspond aux mesures d'interdiffusion de Pimenov, Akkushkarova et Ugaste [8] faites en 1975. Ces mesures constituent en fait une extension en température entre $580^{\circ} \mathrm{C}$ et $840^{\circ} \mathrm{C}$ des seules mesures antérieures de diffusion dans la phase $\beta$ effectuées par Asundi et West [9] en 1964 entre 646 et $750^{\circ} \mathrm{C}$.

Dans cet intervalle de température, nous constatons (Fig. 1) que les résultats sont très proches. Cependant, de très récentes mesures (1983) d'interdiffusion et de diffusion intrinsèque $D_{\mathrm{Al}}^{*}$ et $D_{\mathrm{Cu}}^{*}$ dues à Romig [10] nous ont amené à réexaminer le comportement en fluage haute température de cet alliage. Cette nouvelle approche est rendue nécessaire par le fait que les mesures d'interdiffusion de Pimenov d'une part et de Romig d'autre part aboutissent à des résultats très différents particulièrement en ce qui concerne les énergies d'activation.

Ainsi, nous présenterons succinctement les techniques et résultats des mesures de Pimenov et Romig puis nos résultats de fluage pour un intervalle de température identique et effectuerons le choix du coefficient de diffusion qui régit le comportement en fluage du $\mathrm{CuAl}(\beta)$.

\section{Coefficients d'interdiffusion dans la phase $\beta$ du $\mathrm{CuAI}$.}

Dans les travaux de Pimenov et de ses collègues [8], la diffusion a été étudiée par la technique de la croissance de la phase $\beta$ à partir des phases $\alpha$ (7 \% en poids d'Al) et $\gamma(16 \%$ en poids d'Al) dans l'intervalle de température $580-840^{\circ} \mathrm{C}$.

La mesure de l'épaisseur de la couche $\beta$ et du profil de concentration au travers de cette phase conduit à l'expression de la diffusion chimique $\tilde{D}$ :

$$
\tilde{D}=1,3 \times 10^{-5} \exp \left(-\frac{27000}{R T}\right) \mathrm{m}^{2} / \mathrm{s} .
$$

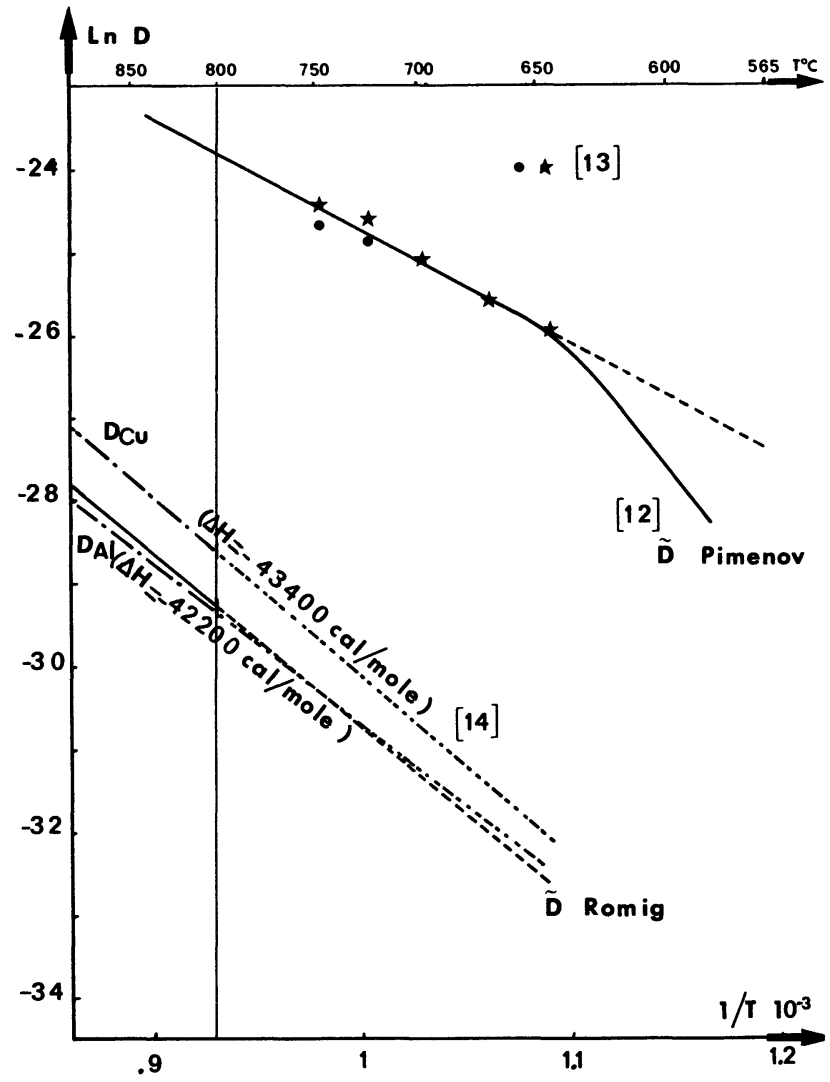

Fig. 1. - Dépendance en température des coefficients de diffusion $\operatorname{Ln} D=f\left(\frac{1}{T}\right)$

[Temperature dependence of diffusion coefficients $\operatorname{Ln} D=$ $\left.f\left(\frac{1}{T}\right)\right]$.

On peut remarquer qu'entre $650^{\circ} \mathrm{C}$ et $565^{\circ} \mathrm{C}$ (température de transition eutectoïde $\beta \rightarrow \alpha+\gamma_{2}$ ), la décroissance de $\tilde{D}$ avec $\frac{1}{T}$ est plus forte que ne le prévoit l'expression (3) (voir Fig. 1). Les auteurs [8] attribuent ce fait à la forte décroissance du facteur thermodynamique $\varphi$, au voisinage de la température de transformation de phase.

Romig [10] étudie le comportement en diffusion de la phase $\beta$ à $800^{\circ} \mathrm{C}, 850^{\circ} \mathrm{C}, 950^{\circ} \mathrm{C}$ en utilisant l'approche expérimentale et la méthode d'analyse de Kirkendal et Darken [11]. Les couples de diffusion ont été construits en se servant de la "Window Frame Technique » et les profils de concentration ont été déterminés par microanalyse électronique [12].

La diffusion chimique $\widetilde{D}$ a été trouvée :

$$
\tilde{D}=6,5 \times 10^{-5} \exp \left(-\frac{42200}{R T}\right) \mathrm{m}^{2} / \mathrm{s}
$$

entre $800^{\circ}$ et $950^{\circ} \mathrm{C}$.

La diffusivité a été observée comme indépendante de la composition sur l'intervalle (11-13\% en poids d'Al). 
Les coefficients de diffusion intrinsèque ont été déterminés :

$$
\begin{aligned}
& D_{\mathrm{A} 1}^{*}=1,3 \times 10^{-5} \exp \left(-\frac{38900}{R T}\right) \mathrm{m}^{2} / \mathrm{s} \\
& D_{\mathrm{Cu}}^{*}=2,2 \times 10^{-4} \exp \left(-\frac{43400}{R T}\right) \mathrm{m}^{2} / \mathrm{s}
\end{aligned}
$$

Les résultats de cette étude sont très éloignés de ceux de Pimenov [8] qui détermine, dans le même intervalle de température, une énergie d'activation de $27000 \mathrm{cal} /$ mole, ce qui est beaucoup plus faible que celle de $42200 \mathrm{cal} / \mathrm{mole}$ obtenue par Romig. L'analyse de Romig est la suivante : les diffusivités mesurées dans les travaux antérieurs apparaissent inconcevablement trop rapides pour un processus de diffusion en volume. Par exemple, il argumente sur le fait qu'au point de fusion à $1050{ }^{\circ} \mathrm{C} \tilde{D}_{\mathrm{Pi}}=4,5 \times 10^{-10} \mathrm{~m}^{2} / \mathrm{s}$ tandis que $\tilde{D}_{\mathrm{Ro}}=6,9 \times 10^{-12} \mathrm{~m}^{2} / \mathrm{s}\left(\tilde{D}_{\mathrm{Pi}}\right.$ et $\tilde{D}_{\mathrm{Ro}}$ mesurées respectivement par Pimenov et Romig) et qu'au liquidus, les diffusivités dans la plupart des métaux cubiques centrés sont approximativement de l'ordre de $10^{-11} \mathrm{~m}^{2} / \mathrm{s}$. La cause de ce désaccord semble inconnue. Ici, il ne s'agit pas pour nous de réaliser une critique des différentes méthodes utilisées mais de choisir quel coefficient d'interdiffusion $\tilde{D}$ est le plus approprié pour traduire le comportement en fluage de notre alliage.

\section{Fluage de la phase $\beta$ du CuAl.}

L'évolution du module de cisaillement avec la température a été obtenue dans la littérature à partir des mesures du module d'Young $(G=0,4 E)$ [13] et par mesure de résonance en vibration forcée autour d'une fréquence de quelques centaines de Hertz [3]. Les valeurs obtenues sont en accord avec celles disponibles dans la littérature.

Pour le fluage, la gamme de température couverte précédemment ( 570 à $630^{\circ} \mathrm{C}$ [4]) a été étendue jusqu'à $855^{\circ} \mathrm{C}$ pour les besoins de ce travail. La dynamique de contrainte compatible avec la technologie de la machine s'étend de 0,2 à $1,6 \times 10^{7} \mathrm{~N} / \mathrm{m}^{2}$ par pas de $0,2 \times 10^{7} \mathrm{~N} / \mathrm{m}^{2}$. Les vitesses de déformation rationnelle $\dot{\varepsilon}_{\mathrm{s}}$ qui en résultent s'échelonnent sensiblement de $1,6 \times 10^{-5} \mathrm{~s}^{-1}$ à $0,15 \mathrm{~s}^{-1}$.

Afin de s'affranchir des effets dus à la cinétique assez lente de la transformation $\left(\alpha+\gamma_{2}\right) \rightarrow \beta$ (existence de fluage inverse pendant toute la durée de l'essai [4]), les éprouvettes sont maintenues trois heures à la température de l'essai avant la mise en charge.

Ainsi, conformément aux résultats relatifs aux phases $\beta$, les courbes $\bar{\varepsilon}=f(t)$ ne présentent pas de fluage primaire mais possèdent un domaine stationnaire très étendu.

Le tracé de $\operatorname{Ln} \dot{\varepsilon}_{\mathrm{s}}$ en fonction de l'inverse de la température $\left(\frac{1}{T}\right)$ reporté en figure 2 donne une énergie d'activation de l'ordre de $35000 \mathrm{kcal} / \mathrm{mole}$ pour $650^{\circ} \mathrm{C} \leqslant T \leqslant 855^{\circ} \mathrm{C}$. Cependant, entre $650^{\circ} \mathrm{C}$ et $565^{\circ} \mathrm{C}$ (température de transition), la décroissance de Ln $\dot{\varepsilon}_{\mathrm{s}}$ avec $\frac{1}{T}$ est plus forte que précédemment.

Cette constatation expérimentale est à rapprocher de celle de l'évolution du coefficient d'interdiffusion $\tilde{D}_{\mathrm{P}_{\mathrm{i}}}$ attribuée à la décroissance anormale du facteur thermodynamique $\varphi$ quand la température tend vers celle de la transition.

Ainsi, quand nous allons opérer le choix du coefficient de diffusion qui régit le comportement en fluage, le facteur $\varphi$ devra être intégré dans l'expression choisie. Ce fait avait été déjà souligné dans des travaux antérieurs $[7,14]$. L'étude de la sensibilité de la vitesse à la contrainte pour différents isothermes conduit à une loi puissance d'exposant $m$ de l'ordre de 3,5, valeur commune aux phases $\beta$ déjà étudiées.

\section{Choix du coefficient de diffusion approprié au fluage.}

Notons tout d'abord que l'énergie d'activation apparente en fluage est encadrée par celle de Romig et Pimenov.

Avec les valeurs explicitées de $D_{\mathrm{Al}}^{*}$ et $D_{\mathrm{Cu}}^{*}$ et la mesure du coefficient d'interdiffusion $\tilde{D}_{\mathrm{Ro}}$ de Romig, trois écritures du coefficient de diffusion [15] intégrant $\varphi$ sont possibles :

a) le coefficient d'interdiffusion $[16,17]$ traduisant généralement le glissement visqueux des dislocations :

$$
\tilde{D}=\varphi\left(C_{\mathrm{Cu}} D_{\mathrm{Al}}^{*}+C_{\mathrm{Al}} D_{\mathrm{Cu}}^{*}\right)
$$

$\left(C_{\mathrm{Cu}}\right.$ et $C_{\mathrm{Al}}$ : fractions atomiques des constituants dans l'alliage $\mathrm{CuAl} ; C_{\mathrm{Cu}}=\frac{3}{4}$ et $\left.C_{\mathrm{Al}}=\frac{1}{4}\right)$;

b) en accord avec Brebec et Poirier [18], si la composition de l'alliage reste homogène, on doit avoir :

$$
D^{\prime}=\varphi\left(C_{\mathrm{Al}} D_{\mathrm{Al}}^{*}+C_{\mathrm{Cu}} D_{\mathrm{Cu}}^{*}\right) ;
$$

c) par contre, selon ces mêmes auteurs [18], si un état stationnaire est atteint, $D$ doit être :

$$
\bar{D}=\varphi\left(\frac{D_{\mathrm{Al}}^{*} D_{\mathrm{Cu}}^{*}}{C_{\mathrm{Al}} D_{\mathrm{Cu}}^{*}+C_{\mathrm{Cu}} D_{\mathrm{Al}}^{*}}\right)
$$

Les mesures de Romig ont été extrapolées jusqu'à $630^{\circ} \mathrm{C}$ (voir Fig. 1) alors qu'en-dessous, les mesures de Pimenov n'étant plus linéaires en $\frac{1}{T}$, l'expression comparative n'a plus de sens. Le report des points dans les coordonnées réduites de Dorn (Fig. 3) avec $\tilde{D}$ fait apparaître une droite de pente 3,4 et d'ordonnée à l'origine telle que $A$ de l'ordre de 1600 .

Il faut signaler une forte dispersion pour les basses contraintes et $750^{\circ} \mathrm{C} \leqslant T \leqslant 855^{\circ} \mathrm{C}$, c'est-à-dire paradoxalement dans l'intervalle de mesure de Romig, avec une tendance à obtenir des droites isothermes parallèles. 


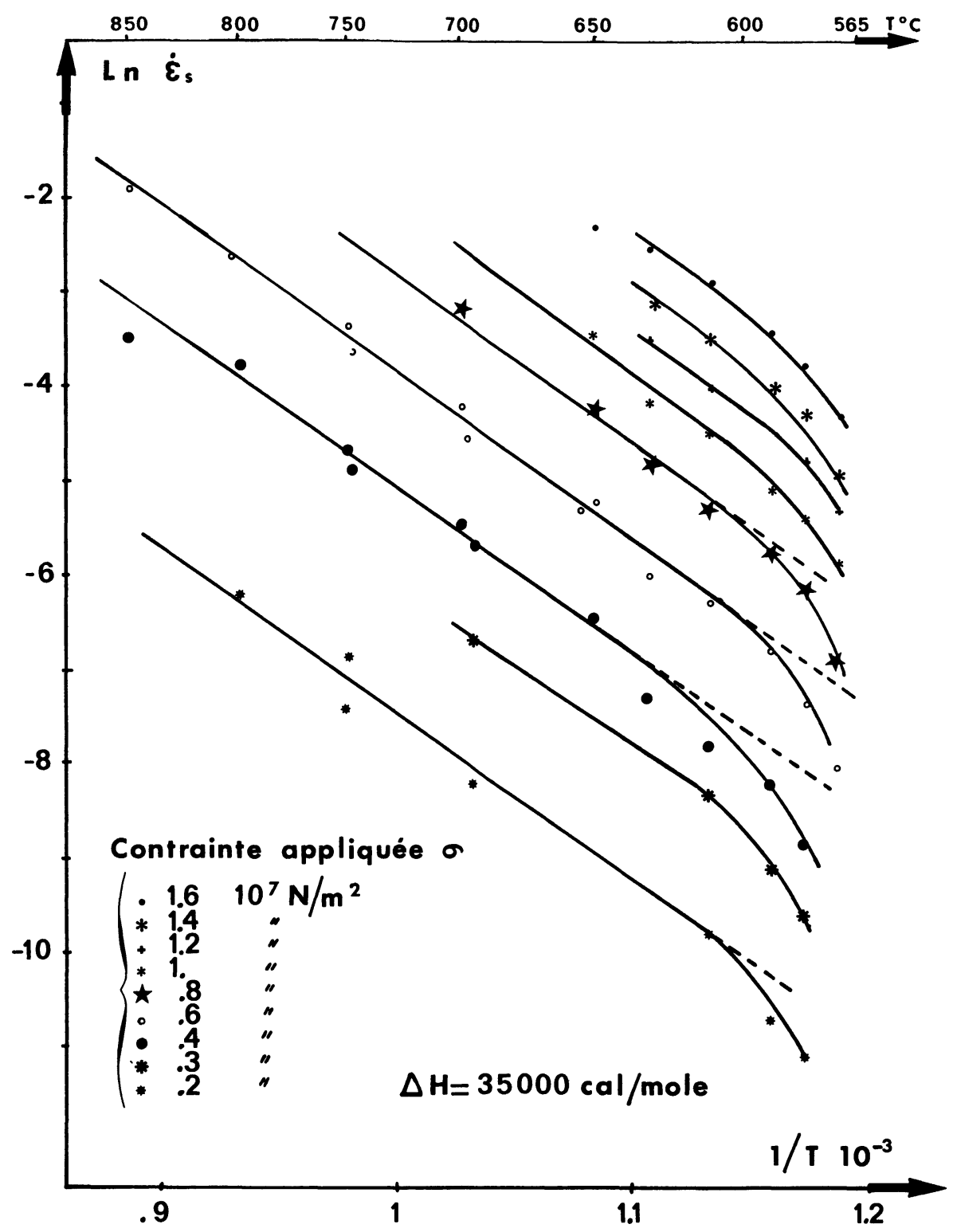

Fig. 2. - Diagramme d'Arrhénius Ln $\dot{\varepsilon}_{\mathrm{s}}=f\left(\frac{1}{T}\right)_{\sigma}$ pour $0,2 \leqq \sigma \leqq 1,6 \times 10^{7} \mathrm{~N} / \mathrm{m}^{2}$.

[Arrhenius diagram $\operatorname{Ln} \dot{\varepsilon}_{\mathrm{s}}=f\left(\frac{1}{T}\right)_{\sigma}$ for $\left.0.2 \leqq \sigma \leqq 1.6 \times 10^{7} \mathrm{~N} / \mathrm{m}^{2}.\right]$

Une régression linéaire effectuée sur 34 points de mesure donne les résultats suivants sur les paramètres de l'équation de Dorn :

$$
\operatorname{Ln} \frac{\dot{\varepsilon}_{\mathrm{s}} k T}{D G b}=\operatorname{Ln} A+n \operatorname{Ln} \frac{\sigma}{G}
$$

avec $r$ coefficient de corrélation.

$\begin{array}{cccc}D & \tilde{D} & D^{\prime} & \bar{D} \\ \bar{n} & \overline{3} & \overline{3,53} & \overline{3}, 47 \\ A & 1600 & 4300 & 2640 \\ r & 0,983 & 0,970 & 0,985\end{array}$

Ce tableau montre qu'il n'est pas possible d'opérer un vrai choix de coefficient de diffusion comme cela avait été possible pour l'AgZn $(\beta)$ [3].

La même représentation avec le coefficient d'interdiffusion de Pimenov (Fig. 4), conduit indépendamment de la contrainte et de la température $\left(570^{\circ} \mathrm{C}\right.$ $855^{\circ} \mathrm{C}$ ) à une droite unique. Une régression linéaire sur 76 points de mesure donne $n=3,41$ avec $A$ de l'ordre de 5 et $r=0,991$.

L'ensemble des valeurs numériques reporté ci-dessus est à confronter avec l'analyse d'Ashby et ses collègues $[19,20]$ qui montrent que l'équation de Dorn n'a un sens physique que seulement si $A$ est voisin de l'unité. 


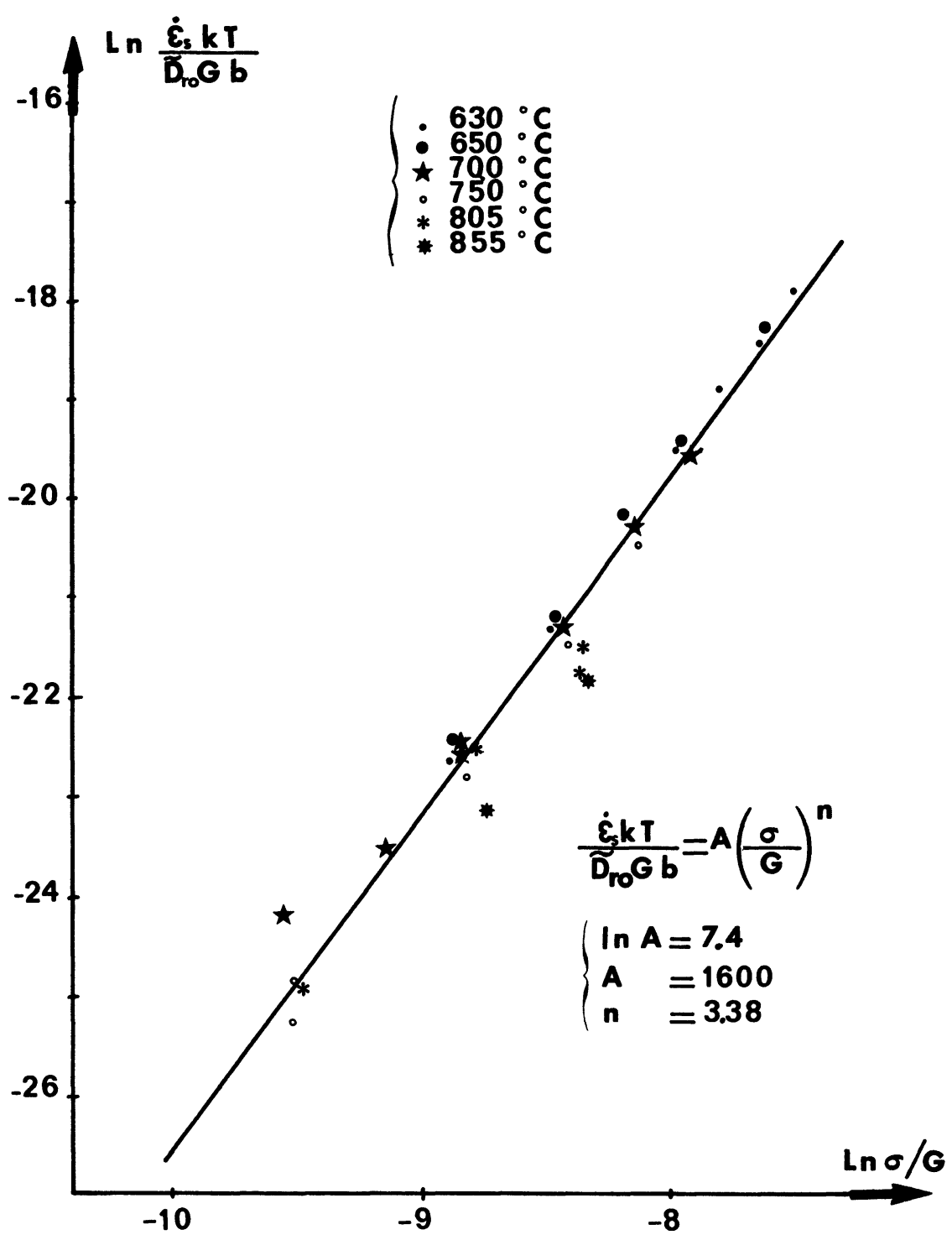

Fig. 3. - Diagramme de Dorn en se servant du coefficient d'interdiffusion de Romig $\left(\tilde{D}_{\mathbf{R o}_{0}}\right)$.

[Dorn diagram when using the interdiffusion coefficient of Roming $\left(\tilde{D}_{\mathrm{Ro}_{0}}\right)$.]

De surcroît, à partir d'une compilation de multiples résultats expérimentaux, ils montrent que l'on obtient :

$$
n=3+0,13 \operatorname{Ln} A
$$

ce qui donnerait :

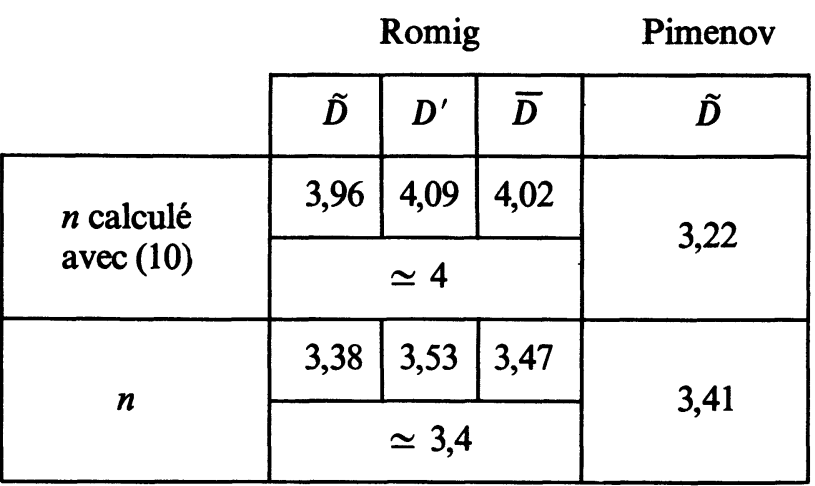

REVUE DE PHYSIQUE APPLIQUERE. - T. 20, № 8, AOOT 1985
En conclusion, nos résultats montrent que l'utilisation des mesures de Romig conduit à :

- une dispersion des points expérimentaux dans le tracé de l'équation de Dorn à haute température (Fig. 3);

- une forte valeur de $A(\sim 1600)$ et de $n(\simeq 4)$ en désaccord avec le comportement "habituel " des phases $\beta$ jusqu'alors étudiées $[2,7]$.

\section{Conclusion.}

Cette analyse sur le choix du coefficient de diffusion pour le comportement en fluage du $\mathrm{CuAl}(\beta)$ permet d'approfonidir la connaissance des mécanismes de diffusion qui régissent les phases $\beta$.

En effet, il semblerait que les mesures de Romig, avec 


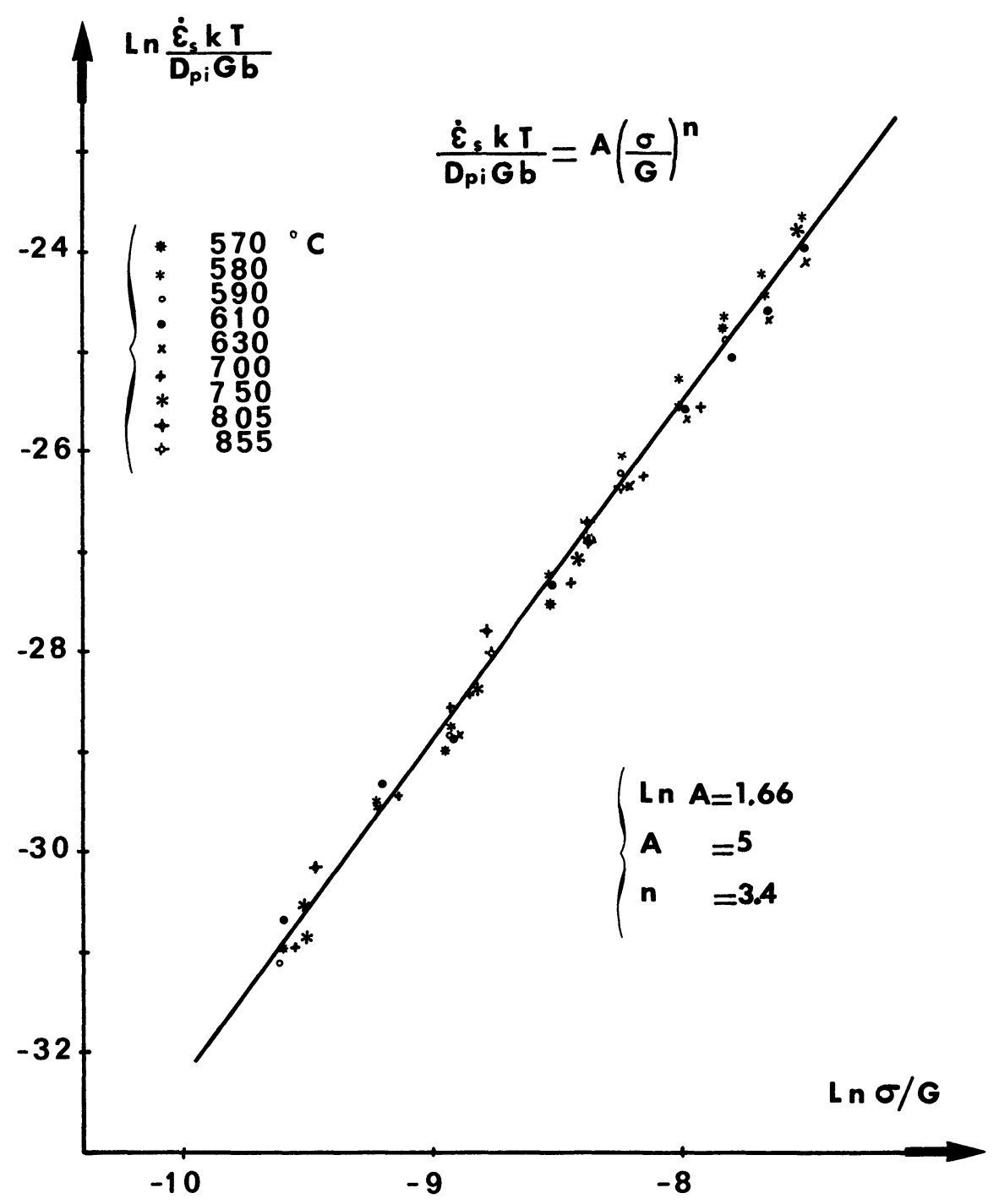

Fig. 4. - Diagramme de Dorn en se servant du coefficient d'interdiffusion de Pimenov $\left(\tilde{D}_{\mathrm{Pi}_{\mathrm{i}}}\right)$.

[Dorn diagram when using the interdiffusion coefficient of Pimenov $\left(\tilde{D}_{\mathrm{Pi}_{\mathrm{i}}}\right) \cdot$ ]

une forte énergie d'activation traduisent bien la diffusion en volume et pourtant ne conviennent pas pour le fluage de cet alliage. L'interprétation classique selon laquelle le fluage à haute température est activé par la diffusion en volume ne semble pas s'appliquer présentement. Le comportement thermomécanique de cet alliage est par contre correctement traduit par le coefficient d'interdiffusion mesuré par Pimenov, avec une énergie d'activation nettement plus faible (de l'ordre des $\frac{2}{3}$ de l'énergie mesurée par Romig). Le fluage serait ainsi contrôlé par des mécanismes de court-circuit de diffusion (tel qu'au cour des dislocations, le long des joints de grain ou des sous-joints de polygonisation, etc...).

Enfin, les mesures de diffusion et de fluage établissent clairement la nécessité d'intégrer le facteur thermodynamique dans l'écriture du coefficient de diffusion.

\section{Bibliographie}

[1] PoIrIER, J. P. - Plasticité à haute température des solides cristallins (Ed. Eyrolles, Paris) 1976.

[2] Bornier, L., Delobelle, P, LeXCellent, C, Mermet, A. and Oytana, C., Metall. Trans. A. 10A (1979) 1944-1947.
[3] Lexcellent, C., Thesis, Besançon, France (1977).

[4] Bornier, L., Delobelle, P. and Oytana, C., Mat. Sci. Eng. 34 (1978) 13-23.

[5] Ahlquist, C. N. and Nix, W. D., Scr. Metall. 3 (1969) 679-682. 
[6] GibBs, G. B., Philos. Mag. 12 (1966) 317-29.

[7] Delobelle, P., Oytana, C., Phys. Status Solidi 75 (1983) 625-634

[8] Pimenov, V. N., Akkushkarova, K. A. and Ugaste, Yu E., Fiz. Met. Metall. 39, no 4 (1975) 821.

[9] Asundi, M. K., WeSt, D. R. F., J. Inst. Met. 92 (1964) 428.

[10] Romig Jr., A. D., J. Appl. Phys. 54, no 6 (1983) 31723175.

[11] DARKEN, L., Trans. AIME 174 (1948) 184.

[12] Goldstein, J. J., De Newbury, Echlin, P., Joy, D. C., FIORI, C. and LIFSHIN, E., Practical scanning electron microscopy and X-Ray microanalysis (Plenum, New York) 1981.

[13] Cabarat, R., Guillet, L. and le Roux, R., C.R. Hebd. Séan. Acad. Sci. 231 (1950) 1373-77.
[14] Delobelle, P., Lexcellent, C. and Oytana, C., Phys. Status Solidi (a) 61 (1980) 701-708.

[15] Crank, J., The mathematics of diffusion (Oxford University, London) 1975.

[16] ONISHI, M., SHIMOZAKI, T. dans DIMETA-82, 405-408. Diffusion and Defect Monograph Series $\mathrm{n}^{\circ} 7$ (1983) (Trans. Tech. Publications. Switzerland, F.R.G., U.K.-U.S.A.).

[17] Guy, A. G., Orkawa, H., J. Non Equilib. Thermodyn. 8 (1983) 159-182.

[18] BrebeC, G., PoIrier, J. P., Acta Metall. 23 (1975) 125.

[19] Stocker, R. L., AshBY, M. F., Scr. Metall.7(1973) 115.

[20] Brown, A. M., AshBY, M. F., Scr. Metall. 14 (1980) 1297. 